\title{
ИЗУЧЕНИЕ ПИКОСЕКУНДНОЙ КИНЕТИКИ ИЗЛУЧЕНИЯ ФРАГМЕНТОВ ХЛОРОПЛАСТОВ С ПОМОЩЬЮ СИНХРОННО НАКАЧИВАЕМОГО ЛАЗЕРА НА КРАСИТЕЛЕ И СПЕКТРОХРОНОГРАФА
}

\section{Введение}

Известно, что при достаточно низкой интенсивности возбуждения около $3 \%$ поглощенного фотосинтезирующими системами света переизлучается в виде флуоресценции за несколько наносекунд. Поэтому наблюдение за кинетикой затухания флуоресценции in vivo комплексов хлорофилла после импульсного возбуждения оказалось весьма плодотворным для изучения разных аспектов процессов фотосинтеза. Начиная с 1969 г. в этих исследованиях широко используются пикосекундные лазеры и соответствующая скоростная регистрирующая аппаратура. Хотя благодаря им и достигнут существенный успех в понимании некоторых особенностей первичных процессов передачи и преобразования энергии при фотосинтезе, возможности пикосекундной флуорометрии еще далеко не исчерпаны. Совершенно новые перспективы открывает здесь сочетание высокого спектрального и временного разрешения на базе нового поколения лазеров и регистрирующей аппаратуры с использованием электронно-оптических преобразователей (ЭОП).

Анализ литературы и экспериментальный опыт показывают, что время затухания свечения $\tau$ хлоропластов и их фрагментов является функцией многих параметров:

$$
\tau=\tau(\mathrm{A}, \mathrm{Б}, \mathrm{B}, \Gamma, \ldots \text { ?), }
$$

где А - объект исследования и его состояние (здесь особенно важны следующие аспекты: метод приготовления, среда разбавления, температура, состояние фотохимического аппарата и т. д.);

Б - условия возбуждения (интенсивность, длительность и спектральный состав возбуждения и т. д.);

В - условия регистрации (спектральный состав излучения, наличие паразитных свечений и т. д.);

Г - метод регистрации (здесь подразумеваётся, что систематические ошибки и неопределенность результатов, присущие разным методам, отличаются).

K сожалению, до сих пор серьезное внимание уделяли только некоторым из этих факторов, в частности в связи с пикосекундными исследованиями интенсивности возбуждения.

Целью данной работы было изучить затухание излучения фрагментов хлоропластов, учитывая по возможности весь комплекс факторов, могущих повлиять на результаты измерений. Важной особенностью при этом является использование пикосекундного спектрохронографа, одновременно разлагающего свечение по спектру и во времени. 


\section{Объекты и экспериментальная установка}

Хлоропласты выделяли из проростков гороха; субхлоропластные фрагменты (обогащенные фотосистемами ФС I или ФС II) получали по методике $\left[{ }^{1}\right]$. Объекты - полусухие тонкие пленки на стеклянной подложке - готовили так же, как в $\left[{ }^{2}\right]$. Измерения проводили при комнатной температуре и при $T=77 \mathrm{~K}$.

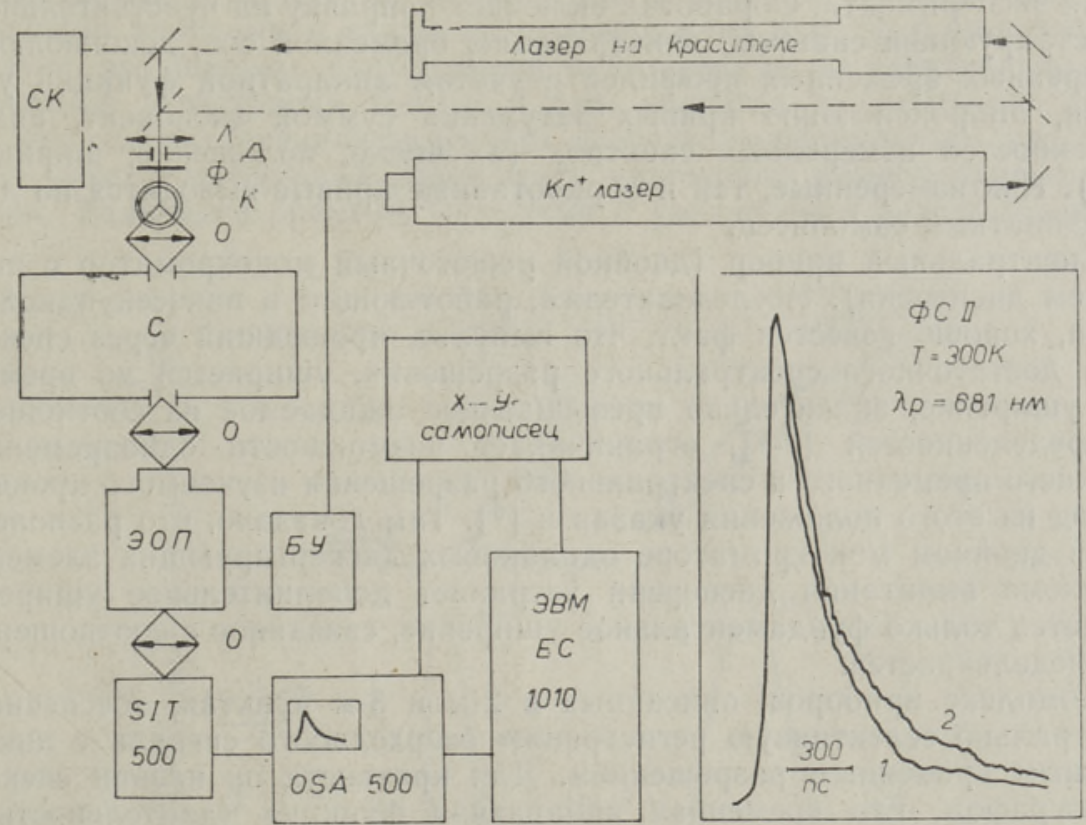

Рис. 1. Схема экспериментальной установки: БУ - блок управления ЭОП, $\mathrm{C}$ - двойной спектрометр с вычитанием дисперсии (собран на базе двух МдР-2), Л - линза, Д - диафрагма, Ф- фильтр, К - криостат, О - объектив, СК - система контроля качества работы лазеров. На вставке кривая затухания флуоресценции ФС II. Возбуждение 647 нм. 1 - измеренная кривая, 2 - она же с поправкой на чувствительность аппаратуры.

Әкспериментальную установку $\left[{ }^{3,4}\right]$ (рис. 1) можно условно разбить на три части:

1. Пикосекундные квазинепрерывные (с частотой следования импульсов $82 M \Gamma$ и) лазеры возбуждения (фирма «Spectra Physics», CША), включающие $\mathrm{Kr}^{+}$-лазер с активной синхронизацией мод (длительность импульса $\sim 75$ nc, пиковая мощность $\sim 100$ Bт) и синхронно накачиваемый лазер на красителе оксазин 1 (пределы генерации 690-805 нм, длительность импульса $\sim 1 n c$, пиковая мощность $\sim 100 \mathrm{BT})$.

2. Регистрирующая система на основе времяанализијрующего ЭОП с видиконным считыванием информации с выходного экрана (система OSA 500 фирмы «B\&M Spectronik», ФРГ) и өбработкой данных на ЭВМ EC 1010 .

Особенностью работы примененного нами ЭОП (УМИ-9ЗШ или УМИ-93М) по сравнению с обычно используемыми является высокочастотный, т. н. синхронный режим развертки $\left[{ }^{5}\right]$, позволяющий регистрировать изучаемое явление во время каждого возбуждающего импульса, т. е. с частотой $82 M Г ц$. Интегрирующее действие люминесцентных экранов ЭОП позволяет в этом режиме изучать сверхслабые (интенсивностью порядка 10 фотон/с) сигналы с высоким (свыше 3 поряд- 
ков) линейным динамическим диапазоном й высокйм отношением сигнал/шум [ $\left.{ }^{4}\right]$. Это снимает необходимость использования в пикосекундных исследованиях сверхмощного возбуждения, что значительно расширяет его возможности при изучении объектов, нуждающихся в деликатном обращении, например, живых клеток, фотосистем и т. д.

Канал связи оптического анализатора OSA 500 с ЭВM EC 1010 обеспечивает оперативный двухсторонний переброс данных (500 каналов за 8 c) и необходимую обработку с. визуализацией на дисплее во время эксперимента. Обработка включает поправку на чувствительность регистрирующей системы, линеаризацию временной оси, деконволюцию измеренных временных профилей с учетом аппаратной функции установки, аппроксимацию кривых затухания суммой экспонент, анализ особенностей измеренных спектров (их число, положение, ширина и т. д.). Как измеренные, так и обработанные кривые выводятся на двухкоординатный самописец.

3. Спектральный прибор (двойной решеточный монохроматор с вычитанием дисперсии). Исследователям, работающим в пикосекундной области, хорошо известен факт, что импульс, прошедший через спектрометр достаточного спектрального разрешения, уширяется во времени. Это уширение, значительно превышающее ожидаемое из соотношения неопределенностей $\left[{ }^{4,6}\right]$, ограничивает возможности одновременного хорошего временного и спектрального разрешения изучаемого процесса. Выход из этого положения указан в [6]. Там доказано, что расположение в двойном монохроматоре одинаковых диспергирующих элементов по схеме вычитания дисперсии устраняет дополнительное уширение. Остается только фундаментальное уширение, связанное с соотношением неопределенностей.

Комплекс приборов, описанных в 2-м и 3-м пунктах, обеспечивает спектрально селективную регистрацию сверхслабого сигнала с пикосекундным временным разрешением. Для краткости он назван спектрохронографом. Его временная аппаратная функция (длительность на полувысоте интенсивности) с УМИ-93Ш - 52 и 89 пс соответственно с импульсами лазера на красителе и $\mathrm{Kr}^{+}$-лазера.

\section{Результаты и обсуждение}

Результаты измерения времен затухания флуоресценции фрагментов хлоропластов в зависимости от интенсивности возбуждения $I$ и длины волны излучения $\lambda_{p}$ при разной длине волны возбуждения $\lambda_{\mathrm{B}}$ и температуры $T$ приведены на рис. 2 и в таблицах. Кривые затухания аппроксимировались суммой двух экспонент с учетом аппаратных искажений.

Таблица 1

Время затухания флуоресценции частиц ФС I при возбуждении на $\lambda_{\mathrm{B}}=647 \boldsymbol{\mu м}$ в зависимости от $\lambda_{p}, I$ и $T, n c$

\begin{tabular}{|c|c|c|c|c|c|c|}
\hline \multirow{2}{*}{ 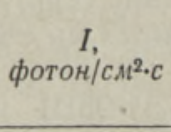 } & \multirow{2}{*}{$\begin{array}{c}I, \\
\text { фотон/См².uмn }\end{array}$} & \multirow{2}{*}{$\begin{array}{l}T, \\
\mathrm{~K}\end{array}$} & \multicolumn{4}{|c|}{$\lambda_{p}, \quad$ MM } \\
\hline & & & 720 & 730 & 738 & 750 \\
\hline $1,9 \cdot 10^{17}$ & $2,3 \cdot 10^{9}$ & $\begin{array}{r}300 \\
77\end{array}$ & $\begin{array}{r}210 \\
1940\end{array}$ & $\begin{array}{r}250 \\
2740\end{array}$ & $\begin{array}{r}300 \\
3200\end{array}$ & $\begin{array}{r}280 \\
3850\end{array}$ \\
\hline $\begin{array}{l}5,8 \cdot 10^{17} \\
3,9 \cdot 10^{18}\end{array}$ & $\begin{array}{l}7,1 \cdot 10^{9} \\
4,8 \cdot 10^{10}\end{array}$ & $\begin{array}{l}77 \\
77\end{array}$ & $\begin{array}{l}1830 \\
1870\end{array}$ & $\begin{array}{l}3140 \\
2850\end{array}$ & $\begin{array}{l}3640 \\
3280\end{array}$ & $\begin{array}{l}4010 \\
3580\end{array}$ \\
\hline
\end{tabular}

При ме ч ани е. Погрешность $\pm 10 \%$. 
Для ФС I доля второй экспоненты оказалась ничтожной и в этом случае использовалось одноэкспоненциальное приближение.

Несмотря на некоторый разброс, экспериментальные результаты обнаруживают по крайней мере три четкие зависимости:

1) от длины волны регистрации, в особенности при $77 \mathrm{~K}$,

2) от температуры,

3) от интенсивности возбуждения.

Из первой зависимости, которая для фотосистем с закрытыми реакционными центрами в явном виде обнаружена в $\left[{ }^{2,7}\right]$, с очевидностью следует, что широко обсуждаемый в литературе по спектрально неселективной регистрации вопрос о том, является ли затухание хлоропластов одноэкспоненциальным или многоэкспоненциальным (см., напр., $\left.\left[{ }^{8}\right]\right)$, имеет тривиальный ответ из-за наложения разных кривых затухания. Удлинение времени затухания с увеличением длины волны на-

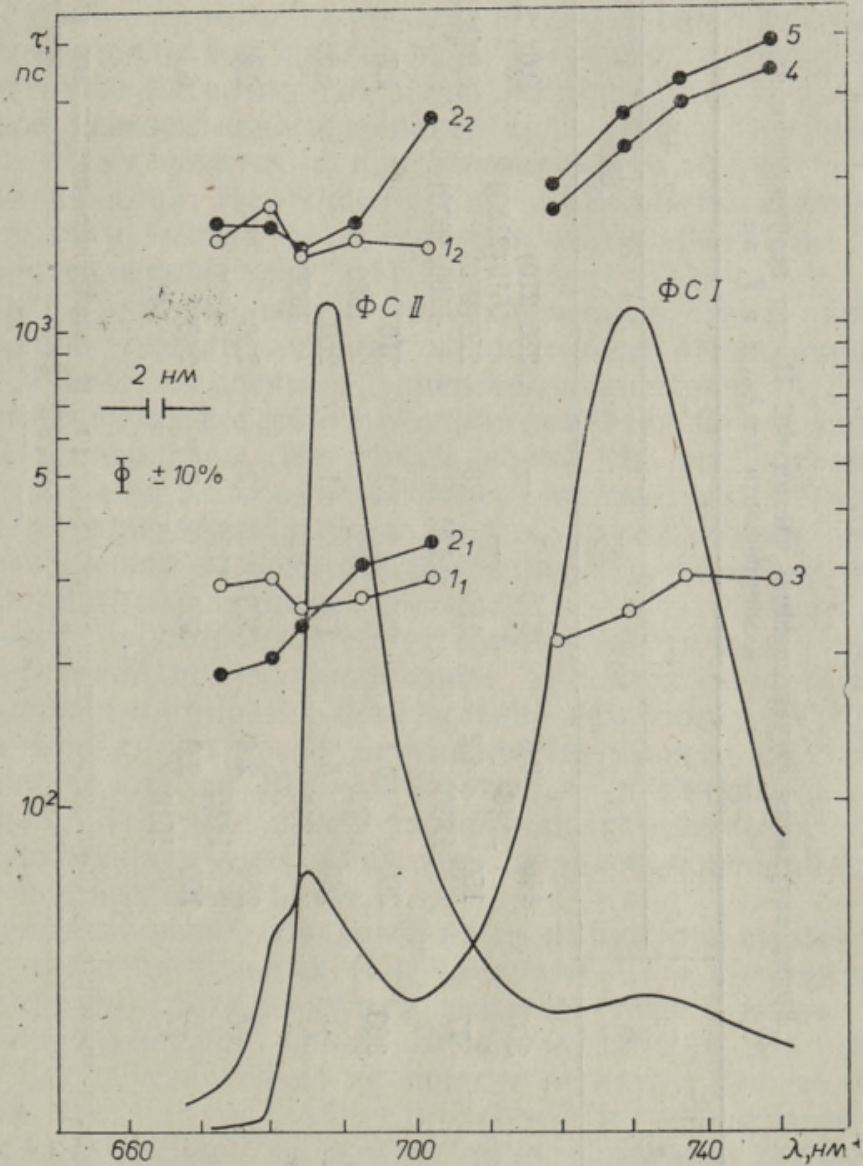

Рис. 2. Зависимость времени затухания флуоресценции ФС I и ФС II от длины волны регистрации при температурах окружающей среды (кривые $\left.1_{1}, 1_{2}, 3\right)$ и $77 \mathrm{~K}\left(2_{1}, 2_{2}, 4,5\right)$. Длина волны возбуждения во всех случаях 647 нм, за исключением кривой 4 , которая получена возбуждением на 703 нм. Интенсивность возбуждения $1,9 \cdot 10^{17}$ фотон/ $\mathrm{cm}^{2} \cdot \mathrm{c}$ для $\Phi$ C I и $5,0 \cdot 10^{18}$ фотон/ $\mathrm{cm}^{2} \cdot c$ для $\Phi C^{\prime}$ II. Приведены также низкотемпературные спектры флуоресценции частиц ФС I и $\Phi$ C II, указана погрешность определения $\tau$ и спектральная ширина щелей спектрометра. 
$\frac{4}{8}$

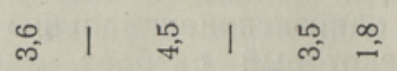

¿

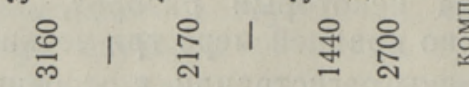

品

$\stackrel{\infty}{-9}$ is ले के

告

के

2

î

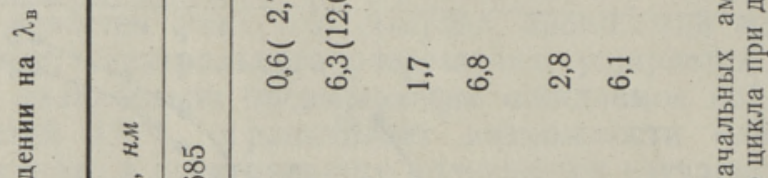

先

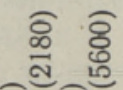

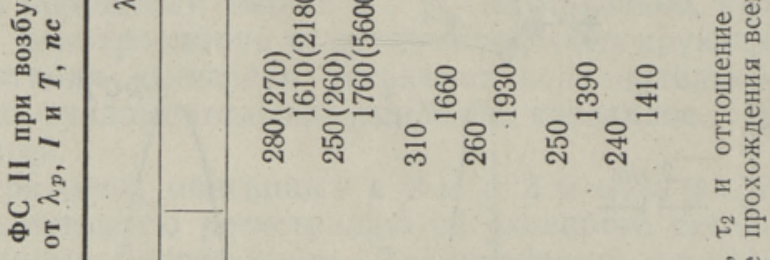

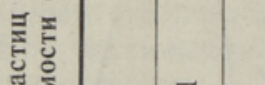

蚛

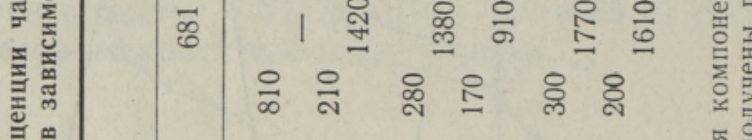

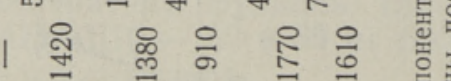

पर

产

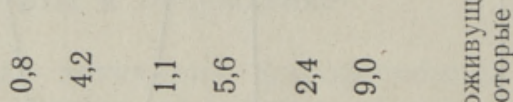

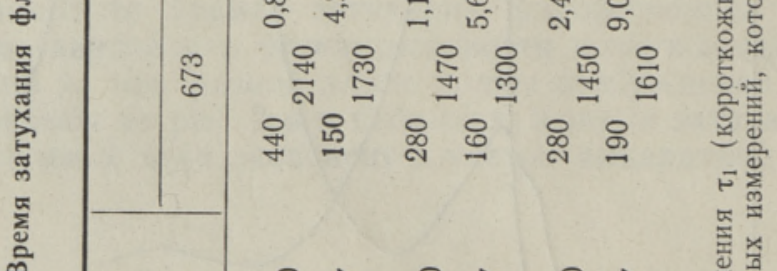

๙

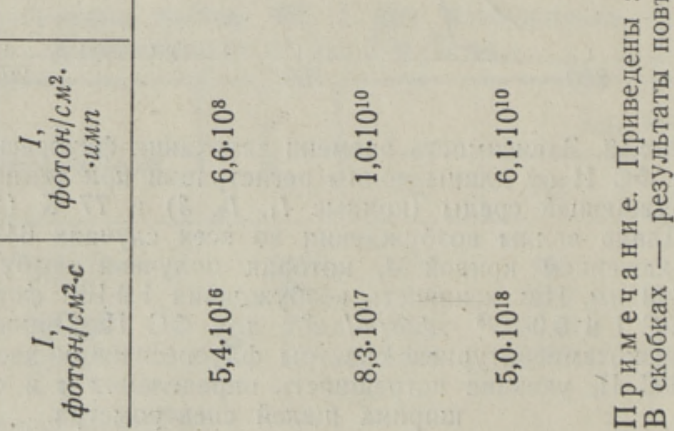


вую интенсивность средняя интенсивность использованного нами возбуждения достаточно высока ** (см. таблицы). Известно, что при непрерывном возбуждении такой интенсивности флуоресценция хлоропластов очень быстро (в течение нескольких секунд или еще быстрее) насыщается из-за закрытия реакционных центров ФС II. Это насыщение проявляется, кроме всего прочего, в существенном увеличении $\tau$.

Сопоставление результатов настоящей работы с полученными на - предварительно облученных объектах (т. е. с заведомо закрытыми реакционными центрами) $\left[{ }^{2,7}\right]$ показывает, что по крайней мере часть центров все же открыта и характеризуется коротким временем $\tau$. Длинная компонента, по-видимому, отражает влияние закрытых центров на эту величину (см. также $[8,11,17])$.

\section{Заключение}

При изучении очень сложных систем, в число которых, несомненно, входят и фотосинтезирующие системы, методические вопросы приобретают первостепенную важность. И вопрос нередко заключается не в том, что исследовать, а в том, как это сделать. На основе результатов данной работы можно утверждать, что наблюдение за спектрально разрешенной пикосекундной кинетикой флуоресценции фотосинтезирующих систем при контролируемой длине волны, интенсивности и частоте следования импульсов возбуждения является весьма перспективным методом, особенно в сочетании с методами пикосекундной абсорбционной спектроскопии. Такой комплексный подход представляется сейчас наиболее обоснованным в данной области.

Авторы благодарны К. К. Ребане за интерес к работе и поддержку, С. М. Кочубей за предоставление объектов, А. В. Райдару, Т. Ю. Хальдре и М. М. Лепик за помощь при обработке результатов на ЭВМ.

** Например, интенсивность излучения Солнца при ясном небе порядка $10^{17}$ фотон/ $\mathrm{CM} \mathbf{M}^{2} \cdot c$.

\section{Л ИТЕР А Т Р А}

1. Островская Л. К.. Кочубей С. М., Рейнгард Т. А., Биофизика, 14, вып. 3, 265-275 (1969).

2. Avarmaa, R. A., Kochubey, S. M., Tamkivi, R. P., FEBS Lett., 102, № $1,139-142$ (1979).

3. Anijalg, A., Freiberg, A., Kaarli, R., Kukk, P., Saari, P., Timp$\mathrm{m}$ an n, K., In: Proc. Symp. «Ultrafast Phenomena in Spectroscopy», Oct. 30Nov. 5, 1980, Reinhardsbrunn. G. D. R., p. 95-99.

4. Аниялг А. О., Берик Е. Б., Вилл А. А., Кукк П. Л., Михкельсоов. Т., С а ари П. М., Ти мпманн К. Э., Фрей берг А. М., В кн.: Материалы II Советско-Французского симпозиума по оптическому приборостроению, 16-21 марта 1981, М., (в печати).

5. Фрейберг А., Р айдару А., Аниялг А., Тимпманн К., Кукк П, С а а р и П., Изв. АН ЭССР, Физ. Матем., 29, № 2, 187-194 (1980).

6. S a a ri, P., Aaviksoo, J., Freiberg, A., Timpmann, K., Opt. Commun., 39, № 1, 2, 94-98 (1981).

7. T a m kivi, R. P., Soovik, T. A., Kochubey, S. M., Avarmaa, R. A. In: Proc. Symp. «Ultrafast Phenomena in Spectroscopy», Oct. $30-$ Nov. 5,1980 , Reinhardsbrunn, G.D.R., p. 350-354.

8. Beddard, G. S., Fleming, G. R.., Porter, G., Searle, G. F. W., S y now i e c, J. A., Biochim. Biophys. Acta, 545, № 1, 165-174 (1979).

9. Butle r, W. L., In: Encyclopedya of Plant Physio!ogy (ed. A. Trebst and M. Avron), new ser., 5, Photosynthesis I, Springer-Verlag, Berlin-Heidelberg, 1977, p. $149-163$.

10. Strasser, R. J., Butler. W. L., Biochim. Biophys. Acta, 462, № 2, 295-306, and $307-313$ (1977).

11. S a uer, K., Brewing to n, G. T., In: Proc. Fourth Intern. Congr. Photosynthesis 1977 (ed. by the Biochemical Society), London, 1978, p. 409-421, 
12. Y u, W., Pellegrino, F., Alf a no, R. R., Biochim. Biophys. Acta, 460, № 1, $171-181$ (1977).

13. Breton, J., Geacintov, N. E., ibid., 594, № 1, 1-32 (1980).

14. Rijgersberg, C. P., Mel is, A., Ames z, J., Swager, J. A., In: Chlorophyll Organization and Energy Transfer in Photosynthesis (ed. bv Excerpta Medica). Ciba Foundation Symp. 61 (new ser.), 1979, p. 305-322.

15. Fink, F., L e u pold, D., Voigt, B., J ü pner, J., Klose, E., Berndt. K., J u n g e, K.. In: Proc. Symp. «Ultrafast Phenomena in Spectroscopy», Oct. $30-$ Nov. 5, 1980, Reinhardsbrunn, G. D. R., p. 356-362.

16. Leupold. D.. Vnigt. B.. Mory, S., Hoffma nn, P., Hieke, B., Biophys. J., 21, № 2, 177-180 (1978).

17. Briantais, J.-M., Merkelo, H., Govindjee, Photosynthetica, 6, № 2, 133-141 (1972).

Ннститут физики

Академии наук Эстонской ССР

A. M. FREIBERG, K. E. TIMPMANN,

R. P. TAMKIVI, R. A. AVARMAA

\section{KLOROPLASTI FRAGMENTIDE KIIRGUSE PIKOSEKUNDILISE KINEETIKA UURIMINE SUNKROONSELT PUMBATAVA VÄRVLASERI JA SPEKTROKRONOGRAAFI ABIL}

Artikkel käsitleb herne kloroplasti fotosüsteemide (FS) I ja II kiirguse pikosekundilist kineetikat toatemperatuuri! ia vedela lämmastiku keemistemperatuuril. Kasutatud on uudset eksperimendiseadet, mis võimaldab saavutada üheaegset kõrget spektraal- ja aeglahutust. On leitud kustumisaegade küllalt suur sõltukus registreeritavast lainepikkusest ja temperatuurist, kusiuures FS II puhul on sõltuvus mittetriviaalne. Ergastuse nõrgale intensiivsusele vaatamata $\left(\sim 10^{10}\right.$ footon/ $\left.\mathrm{cm}^{2} \cdot \mathrm{imp}\right)$ on iälgitav toatemperatuurile vastava FS II kiirguse eluea lühenemine ergastuse istensiivsuse kasvades.

A. M. FREIBERG, K. E. TIMPMANN,

R. P. TAMKIVI, R. A. AVARMAA

\section{INVESTIGATION OF PICOSECOND FLUORESCENCE KINETICS OF CHLOROPLAST FRAGMENTS BY SYNCHRONOUSLY-PUMPED DYE-LASER AND SPECTROCHRONOGRAPH}

Picosecond fluorescence kinetics of pea chloroplast photosystems PS I and PS II was studied at room and LN temperatures. A novel experimental equipment was used which allows to obtain simultaneous high spectral and temporal resolutions. As a result, a remarkably strong decay-time dependence on measuring wavelength and temperature is found, whereas for PS II the dependence is non-trivial. Despite the low exciting intensity $\left(\sim 10^{10} \mathrm{ph} . / \mathrm{cm}^{2}\right.$. pulse $)$ a slight decrease of PS II lifetime with the increase of the exciting intensity is observed at room temperature, 